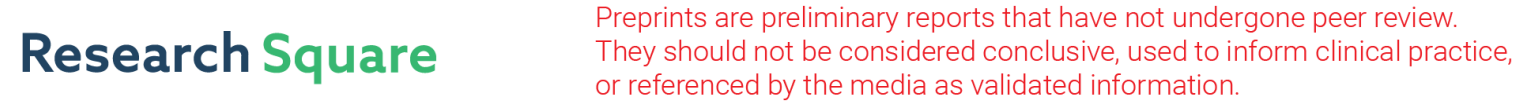 \\ Free-Space Optical Link Optimization in Visible Light Communication System
}

\section{Ngene Chidi Emmanuel ( $\nabla$ chidingene2279@gmail.com )}

University of Johannesburg - Auckland Park Bunting Road Campus https://orcid.org/0000-0003-03086762

\section{Prabhat Thakur}

University of Johannesburg - Auckland Park Bunting Road Campus

\section{Ghanshyam Singh}

University of Johannesburg - Auckland Park Bunting Road Campus

\section{Research Article}

Keywords: Bit error rate (BER), optimization, free space optical communication channel (FSOC), LEDs, visible light communication (VLC),

Posted Date: April 8th, 2021

DOI: https://doi.org/10.21203/rs.3.rs-358149/v1

License: (1) (1) This work is licensed under a Creative Commons Attribution 4.0 International License. Read Full License 


\title{
Free-Space Optical Link Optimization in Visible Light Communication System
}

\author{
C. E. Ngene, P. Thakur, and G. Singh \\ Department of Electrical and Electron cs Engineering Science \\ Auckland Park Kingsway Campus, P.O Box 524. University of Johannesburg. Johannesburg-2006, South Africa \\ e-mail: Chidingene2279@gmail.com,prabhatt@uj.ac.za, ghanshyams@uj.ac.za
}

\begin{abstract}
In this paper, a free-space optical link (FSOL) optimization in the visible light communication (VLC) is performed to improve the channel performance for signal strength and analysze the effect of signal failures in a communication system. The problem of uncertainty in the FSOL components for a better signal has prompted this study to check for an improved and enhance signal using bit error rate (BER) analyzer. The light emitting diode (LED) is used as a carrier for transmission of data as the optimization of system properties have concluded for limitations and working conditions. The noise has been added and removed to ascertain the channel behavior which in turn display an acceptable eye diagram for an improved signal. The acceptable eye diagram shows a better signal in the system. The optimization of FSOL will guarantee multi-mobile users of shared signal which will improve the signal errors, loss of power, weak signal, signal failure, loss of data etc. To fully characterize the FSOL, OptiSystem software tool has been used.
\end{abstract}

Keywords-Bit error rate (BER), optimization, free space optical communication channel (FSOC), LEDs, visible light communication (VLC),

\section{INTRODUCTION}

The development of new technology in optics system has been extended to research on the quality of signal performance in a given channel for visible light communication (VLC) systems. A free-space optical link (FSOL) optimization in VLC is designed using OptiSystem software to improve the channel behavior. A light emitting diode (LED) is used as a carrier for transmission of data as the optimization of the system properties were performed for limitations and working conditions. Noise were added and removed to ascertain the channel behavior which in turn display an acceptable eye diagram for an improved signal. The acceptable eye diagram shows a better signal in the system. The optimization of FSOL will guarantee multimobile users of shared signal which will improve signal errors, loss of power, weak signal, signal failure, loss of data etc. To fully characterize the FSOL, OptiSystem software tool was used. OptiSystem is a designed tool to test, plan and simulate any optical link model that uses optical network such as metropolitan area network (MAN), local area network (LAN), small area network (SAN), wide area network (WAN) and so on, with a broad spectrum. Researchers have modelled the FSO link with other communication link presenting a high signal to noise ratio average rule with the simplest function in [1]. Researchers have studied on the possible way to compensate the signal distortion in FSOL by engaging an optical phase conjugation in [2]. FOSL characteristics have been investigated for its high speed optical interconnection to overcome short range interconnection in a given system [3]. Researchers have used infrared FSO for outdoor communication under weather conditions which provide a solution for a wireless communication using a high capacity [4]. Researchers have compared two techniques for an improved BER performance and recommend one in [5]. The researchers have proposed the use of emitting laser to transmit signals in an optical femtocell link which records a minimum bit error rate within an indoor environment in FSOC [6]. Visible light generated by LED is a promising candidate with benefits such as small power consumption, bandwidth free license channels and so on [7]. The VLC applications in the traffic lights [7], smart homes [8], for construction of an IR based devices for urban health centers [9] and power allocation (PA) techniques for VL-NOMA networks using LED as a carrier [10].

\section{THEORETICAL BACKGROUND}

Free space optical communication (FSOC) transmits data through lighting in a communication set-up. Researchers have proposed the use of spatial and temporal statistics phase features which can be inter-converted for FSOL in [11]. Researchers have used high altitude platforms (HAPs) for high data rate solution with FSOL in [12]. The study of digital coherent combing (DCC) method is to provide high sensitivity signals in minimizing the complexity of the algorithm used for FSOC [13]. The researchers have study the challenges in the FSOL proposing a technique as a solution to overcome them in [14]. Researcher have display an acceptable eye diagram in four-level pulse amplitude modulation (PAM4) analysis with wavelength division multiplexing (WDM) in FSOL [15]. Researchers have studied and evaluate the performance of a special technique used to receive signal in FSO transmission in [16]. Researchers have studied the FSO system for signalling without having channel state information comparing it with conventional receiver within the system [17]. Researchers have experimented and analysed an analogue coherent FSOL for local cloud based radio access system in [18]. The adaptive wireless system is been implemented using FSO method in [19]. Researchers have proposed the use optical wireless communication (OWC) for high traffic, massive connectivity with radio frequency technology in the fifth generation wireless communication [20]. FSO/RF investigates the performance of the amplify and forward multi-hop with the characteristics which includes high speed, availability in poor weather, longer range, low cost, for improved low SNR [21]. OWC can be applied for indoor, underwater, space and outdoor communication especially for data centers and indoor LAN [22-23], in [24] underwater sensing, in [25] space communication, and in [26] mobile networks backhaul. For indoor VLC, power is consumed for lighting and communication in a transmission setting [27]; the amount of power consumed is based on data usage which is subject to the driver circuitry. Researchers have studied an optimal Lambertain order algorithm in [28]. In [29], the researchers studied the AC and DC concept of optical power but did not study the illumination distribution and power consumption optimization. Optical power with data transmission and brightness control is been studied in 
VLC based on energy efficiency in [30]. OptiSystem software is a simulation tool for testing, designing, and optimization of optical link using different components and equipment to visualize optical transmission for analysis [31]. The researcher have proposed the use of RF/VLC and FSOC network to overcome the challenges encountered in RF systems for online social services in [32]. The advantages of FSOL characteristics in atmospheric conditions have improve the iteration optimization which minimizes the BER for better performance [33]. The OptiSystem can be deployed to simulate different applications in the field of telecommunication and beyond. We have carefully designed, tested and simulated an FSO Link in VLC using OptiSystem software. The author's contribution to this paper is summarized as follows:

- We have successfully modelled a free-space optical link using OptiSystem software for an improved signal performance which displays an acceptable eye diagram using BER analyser.

- We have used the vertical axis difference to check the quantity of noise ratio in the system, displaying the problem encountered such as signal error, low power, shortage of power, loss of power, weak signal, loss of data, loss of signal, signal failure etc.
- The realized eye diagram is as a result of the synchronized system model for LED to photodiode nodes communication.

- We have tested an optimize communication link for the optical ranges $(\mathrm{km})$ and optical filter at the system.

- We have added and removed noise from the electronic component to ascertain the behaviour of the signal performance.

- Our accepted eye diagram result guarantees multimobile users of shared signals within the area of transmission which will improve and enhance the system throughput.

- We have employed the BER analyser to display the acceptable eye diagram for the designed system model.

However, this paper opens more research opportunity and doors for researchers for the development and application of the OptiSystem software tool for FSO link in VLC networks in a scientific way. The rest of the papers are sectionalized as follows: Section II discussed the theoretical background. Section III, detailed the simulation set-up. Section IV, presents the experimental results. Section V, narrates the conclusion.

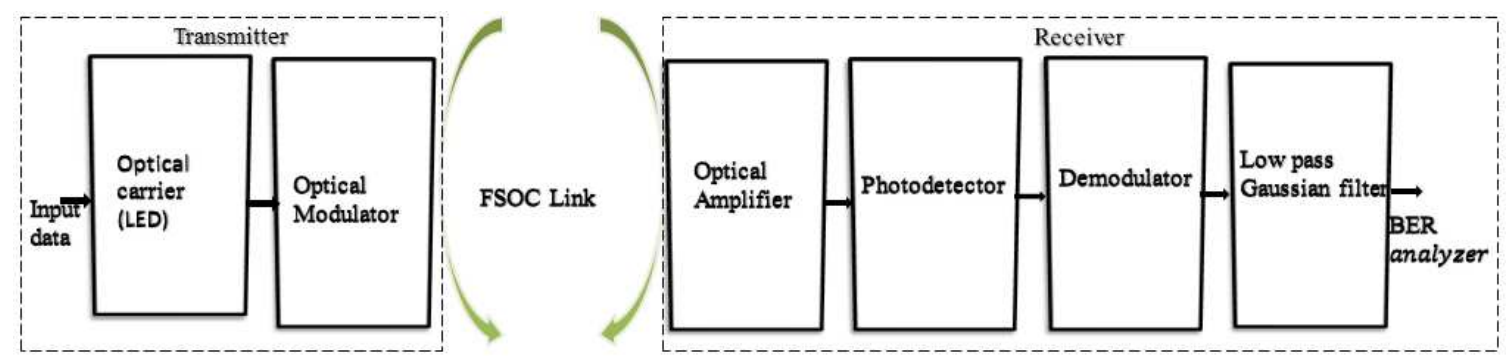

Fig. 1 A block diagram of a free-space optical link optimization in visible light communication system.

The FSOL comprises of the optical carrier (LED), modulator, FSOC link, optical amplifier, optical receiver (photodetector), demodulator, low pass filter and BER analyzer. The optical carrier (LED) transmits data through lightning, the data is modulated, the optical modulator device modulate data through a beam of light as the LED transmits data through lighting, then passes FSO link. FSO link is a wireless means of transmitting data optically in a medium. The optical amplifier amplifies the optical data in FSO link to be detected fully by photodetector. An optical amplifier directly amplify optical signal without converting the signal to electrical, which then get detected by optical receiver (photodetector). The photodetector sense the data through lighting and convert it into current, the received signal is demodulated. The demodulator is retrieving the original data through a carrier wave electronically allowing the low pass Gaussian filter to filter the signal. The low pass filter allows the signal to be passing to a lower or higher frequency depending on the set device cut-off frequency and BER analyzer displays the recovered signals. The BER analyzer is a device that calculates the BER which is as a function of the energy per bit to noise power spectral density ratio displaying the output signal in the form of eye diagram. The data signal transmitted has an electrical signal in the form of 1' and 0's that is the form of a pseudo- random binary bit sequence and non-return to zero coding standards.

\section{SIMULATION SET-UP}

In this section, the designed FSO link in VLC is presented. We have optimized the designed communication system characteristics at the transmitter side, the FSOL and the receiver side to display the acceptable eye diagram. $1.0864 \mathrm{~mW}$ LED power was used to transmit data to the system, from the pseudo-random bit sequence generator $1.5 \mathrm{Gbits}$ generate the bit rate speed at which the data transmits for the accepted opening of the eye diagram. The NRZ pulse generator_ 1 is used for the amplitude, rise time and fall time of the eye diagram. Mach-Zehnder modulator is used to modulate the incoming bits from the LED; the FSO channel is used as a medium of transmission which decides the maximum and minimum range $(\mathrm{km})$ to display the acceptable eye diagram performance within the system. An optical amplifier is used to amplify the weak signal to boost the signal strength for continuous transmission to the receiver side. An optical DPSK receiver is used to receive the signals. The electrical phase demodulator is used to demodulate the data received for better performance, low pass Gaussian filter is used to filter the demodulated bits recovering the original data transmitted. 


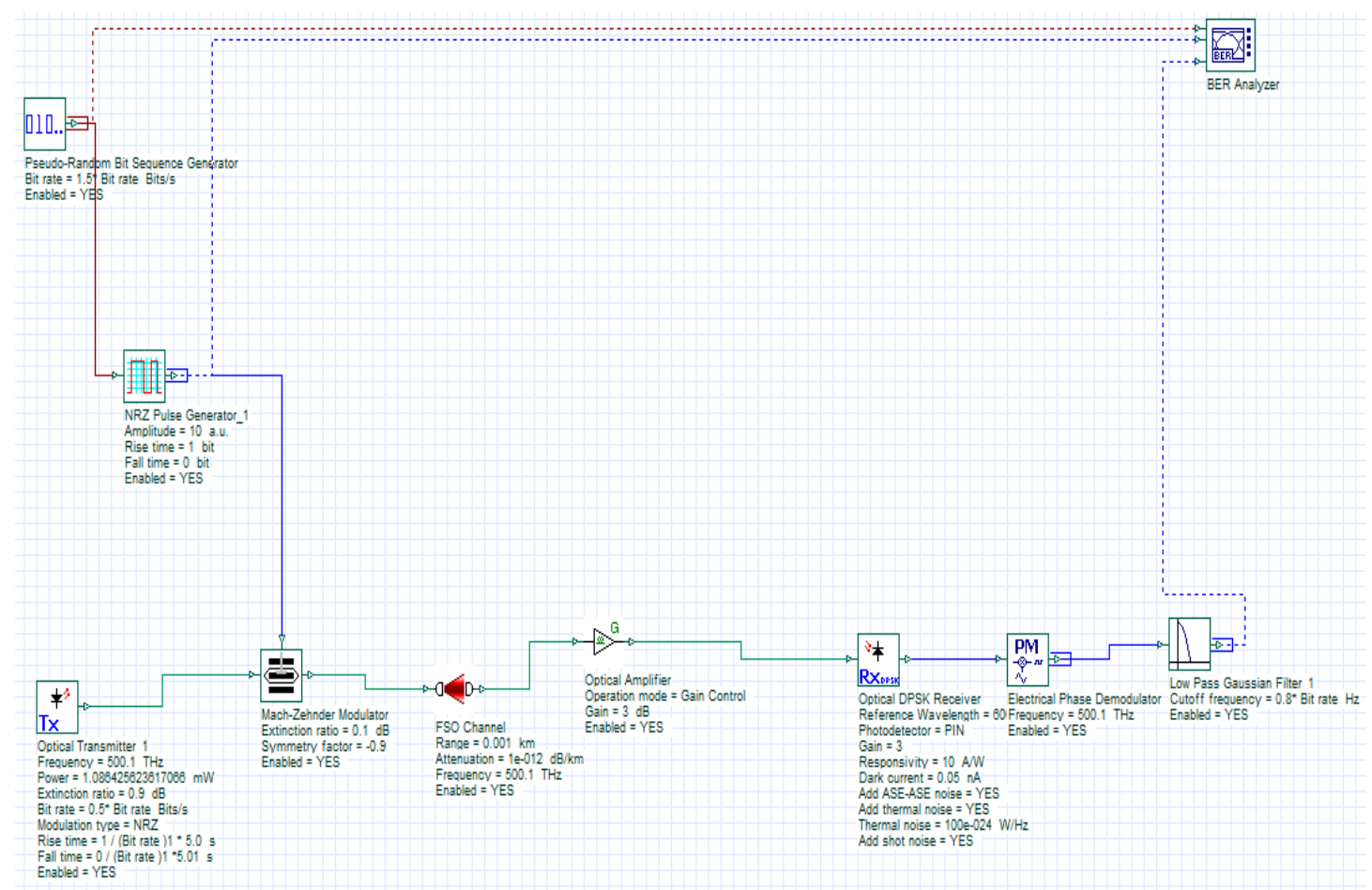

Fig. 2 The system model of a free-space optical link optimization in visible light communication system.

BER analyzer displays the opening and closing of the eye diagram when the system is in operation. The speed at which data is been transmitted and recovered is of outmost important in this paper as $1.5 \mathrm{Gbits} / \mathrm{s}$ has been used to transmit data and 0.3 milliseconds is used to recover the signal by displaying the eye diagram. Noise from the optical amplifier and optical detector were added at different intervals and remove at a certain range $(\mathrm{km})$ to ascertain the closing of the eye diagram, error was observed as the range is been increased.

Table 1 optimize parameters used in the free-space optical link optimization in visible light communication system

\begin{tabular}{|l|l|}
\hline LED input power & $1.0864 \mathrm{~mW}$ \\
\hline LED frequency & $500.1 \mathrm{THz}$ \\
\hline Extinction ratio (LED) & $0.9 \mathrm{~dB}$ \\
\hline Bit rate & $0.5 \mathrm{bit} / \mathrm{s}$ \\
\hline Modulation type & $\mathrm{NRZ}$ \\
\hline Rise time & $1 /($ Bit rate) $15.0 \mathrm{~s}$ \\
\hline Fall time & $0 /($ Bit rate $) 15.01 \mathrm{~s}$ \\
\hline Mach-Zehnder Modulator & $0.1 \mathrm{~dB}$ \\
\hline Extinction ratio & $0.001 \mathrm{~km} \mathrm{to} 0.009 \mathrm{~km} \mathrm{and}$ \\
\hline FSOC channel ranges & $0.01 \mathrm{~km}$ to $0.05 \mathrm{~km}$ \\
\hline Attenuation & $1.2 \mathrm{~dB}$ \\
\hline FSOC Frequency & $500.1 \mathrm{THz}$ \\
\hline Optical amplifier gain & $3 \mathrm{~dB}$ \\
\hline
\end{tabular}

\begin{tabular}{|c|c|}
\hline $\begin{array}{l}\text { Optical DPSK receiver } \\
\text { wavelength }\end{array}$ & $600 \mathrm{~nm}$ \\
\hline Gain & $3 d B$ \\
\hline Responsivity & $10 \mathrm{~A} / \mathrm{W}$ \\
\hline Dark current & $0.05 \mathrm{nA}$ \\
\hline $\begin{array}{l}\text { Electrical phase } \\
\text { demodulator frequency }\end{array}$ & $500.1 \mathrm{THz}$ \\
\hline $\begin{array}{l}\text { Low pass Gaussian filter cut } \\
\text { off frequency }\end{array}$ & $0.8 \mathrm{~Hz}$ \\
\hline $\begin{array}{l}\text { Pseudo-Random bit } \\
\text { sequence generator bit rate }\end{array}$ & 1.5 Bit rate \\
\hline $\begin{array}{l}\text { NRZ Pulse generator_1 } \\
\text { amplitude }\end{array}$ & $10 \mathrm{~m} \cdot \mathrm{s}^{-1}$ \\
\hline
\end{tabular}

\section{RESULTS AND DISCUSSION}

The experimental results are retrieving using OptiSystem software tool. The parameters such as optical LED power, extinction ratio, rise and fall time, bit rate, wavelengths are optimized for system performance. For each parameter change, others were kept as default illustrated in Table 1. The measurement of the eye diagram is evaluated by deducting the double values obtained from the optical signal to noise ratio (OSNR). The eye diagram is used to indicate the quality of signals transmissions. The measurement of the signal is taken without noise and later the noise was added to identify the influence of the noise on the OSNR and the jitter characteristic. The ratio between the eye diagram opening reference value and the ratio of the eye diagram closing values with the influence of noise was calculated and used as an observation. The difference in the ratio of eye diagram calculated is seen in the graph for proper interpretation. 


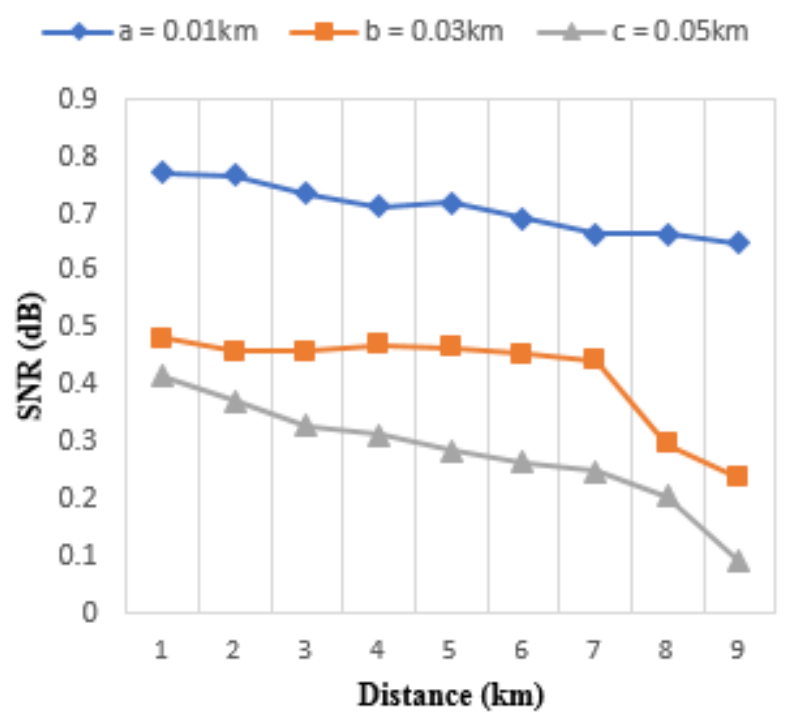

(a)

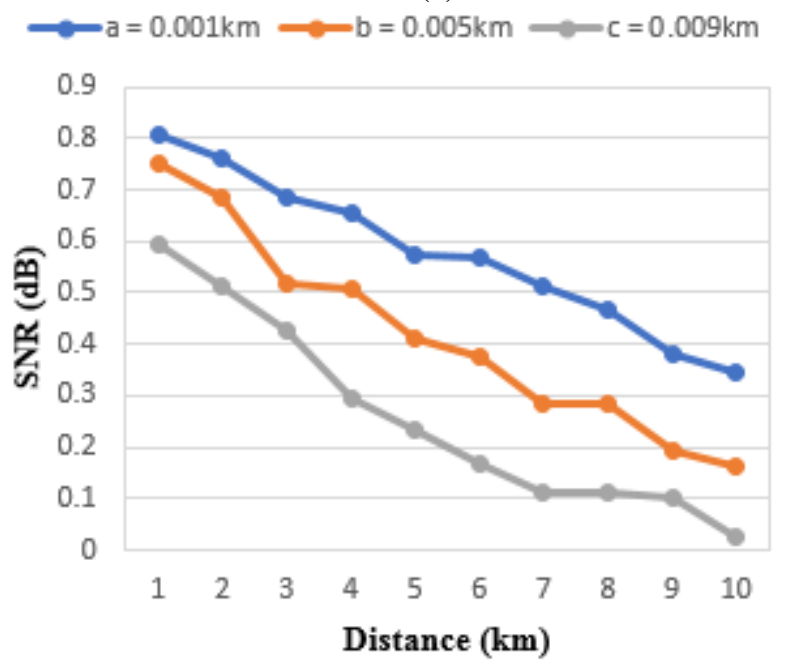

(b)

Fig. 3 Variation on the SNR (in dB) with distance in the indoor noise added ranges for (a) optical amplifier (b) optical detector.

Fig. 3(a) displays the closing of the eye diagram using the BER analyzer, when optical amplifier noise was added at the range of $0.01 \mathrm{~km}, 0.03 \mathrm{~km}$ and $0.05 \mathrm{~km}$. This shows the gradual closing of the eye diagram as the signal gets corrupted by different noise level. At $0.01 \mathrm{~km}$ different noise ratios were added, the vertical axis of the received eye diagram was recorded and the different was used for analysis. We recorded that at $0.79 d B$ a noise of $0.1 \mathrm{~W} / \mathrm{Hz}$ was added up-to $0.9 \mathrm{~W} / \mathrm{Hz}$ subsequently. We observed that the signal to noise ratio drops or decreases to $0.68 d B$. We notice that as the noise was added in the system the signal strength decreases thereby causing the eye diagram to close gradual and signal is lost. The signal to noise ratio drops to $0.68 d B$, this shows that noise is notice in the system but there is still a tradeoff of signal.

At $0.03 \mathrm{~km}$ noise were added, the vertical axis of the received eye diagram was recorded and the different was used for analysis. We observe that at $0.4 d B$ a noise of $0.1 \mathrm{~W} / \mathrm{Hz}$ was added up-to $0.9 \mathrm{~W} / \mathrm{Hz}$ subsequently. We recorded that the signal to noise ratio drops or decreases to
$0.27 d B$. We notice that as the noise was added in the system the signal strength decreases thereby causing the eye diagram to close gradual and signal is lost. The signal to noise ratio drops to $0.27 \mathrm{~dB}$, this shows that noise is notice more in the system, but there is still a little tradeoff of signal.

At $0.05 \mathrm{~km}$ noise were added, the vertical axis of the received eye diagram was recorded and the different was used for analysis. We recorded that at $0.4 d B$ a noise of $0.1 \mathrm{~W} / \mathrm{Hz}$ was added up-to $0.9 \mathrm{~W} / \mathrm{Hz}$ subsequently. We observed that the signal to noise ratio drops or decreases to $0.4 \mathrm{~dB}$. We notice that as the noise was added in the system the signal strength decreases thereby causing the eye diagram to close gradual and signal is lost and an error is observed as the signal is heavily corrupted by noise. The signal to noise ratio drops to $0.02 d B$ this shows that noise is more in the system, hence signal is lost completely.

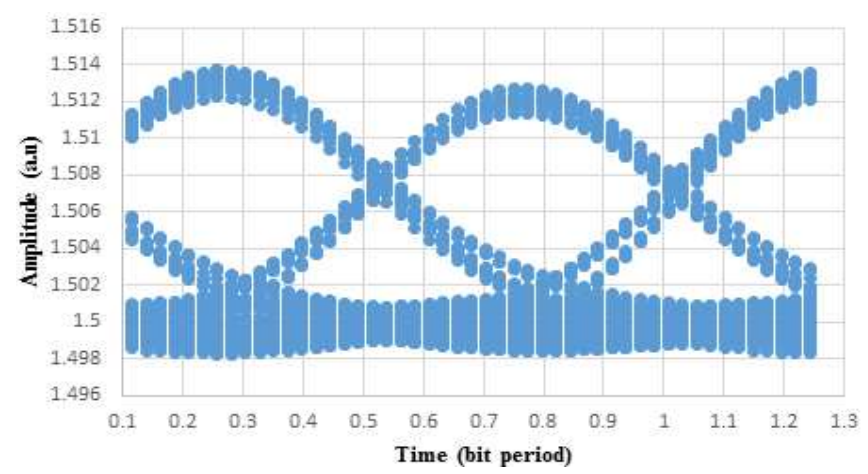

(a)

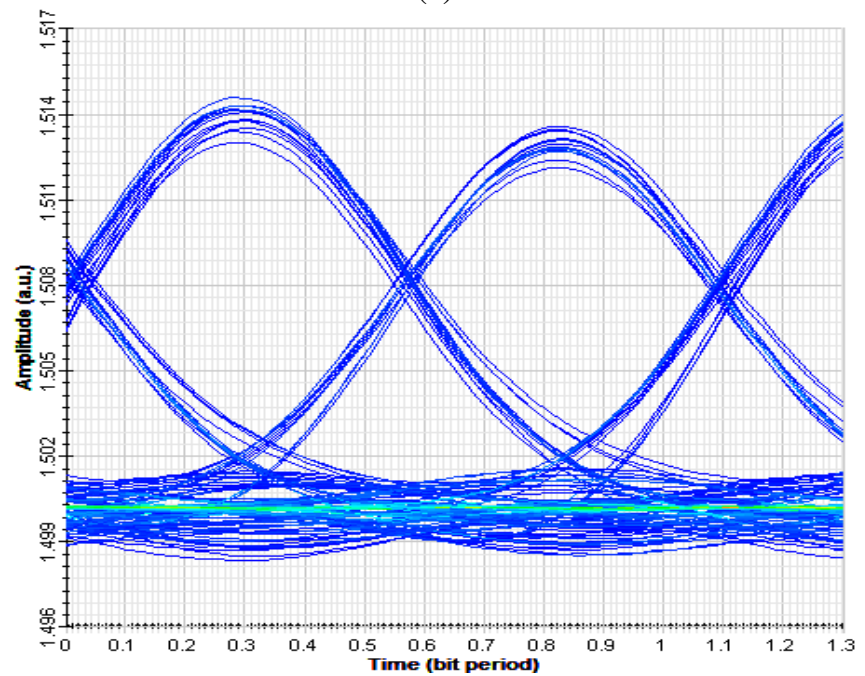

(b)

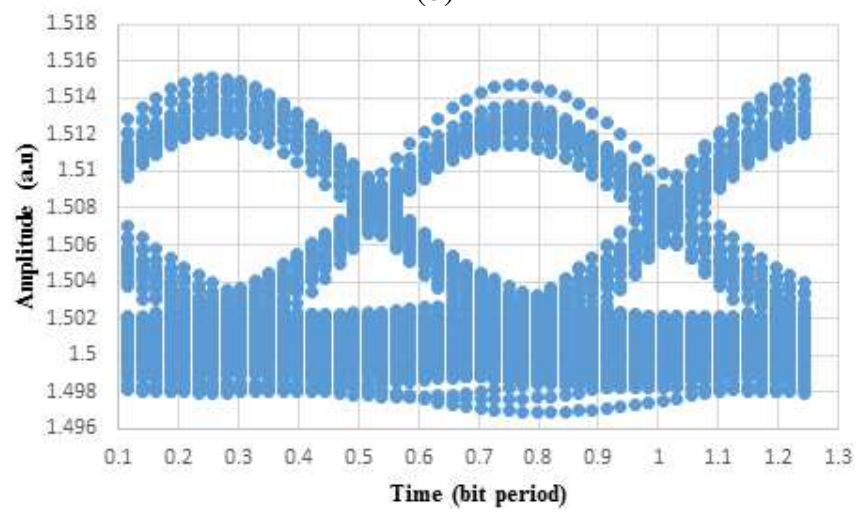

(c) 


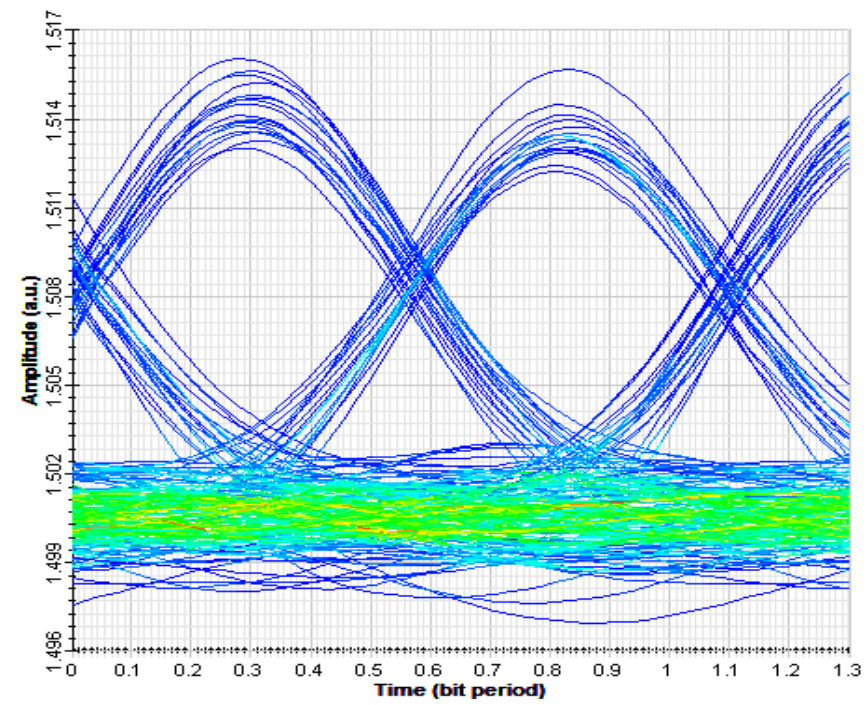

(d)

Fig. 4 The eye diagram using OptiSystem and excel software (a) acceptable eye diagram from excel without noise, (b) acceptable eye diagram from OptiSystem without noise (c) eye diagram with noise from excel (d) eye diagram from OptiSystem with noise.

Fig. 3(b) optical detector analysis, noise is added to see the behavior of the signal in the system throughput using different acceptable eye diagram ranges (distances) from the FSOC link. ASE, distribution (Gaussian), thermal and short noise were added within the range of $0.001 \mathrm{~km}$ to $0.009 \mathrm{~km}$ from the optical detector. At $0.001 \mathrm{~km}$ noise were added, the vertical axis of the received eye diagram was recorded and the different was used for analysis. We observe that at 0.8 $d B$ a noise of $3000 \mathrm{~W} / \mathrm{Hz}$ was added up-to $30000 \mathrm{~W} / \mathrm{Hz}$ subsequently. We recorded that the signal to noise ratio decreases to $0.36 d B$. We notice that as the noise was added in the system the signal strength decreases thereby causing the eye diagram to close gradual and signal is lost. The signal to noise ratio drops to $0.36 d B$ this shows that noise is notice in the system, but there is still a little tradeoff of the signal.

At $0.005 \mathrm{~km}$ noise were added, the vertical axis of the received eye diagram was recorded and the different was used for analysis. We observe that at $017.8 \mathrm{~dB}$ a noise of $3000 \mathrm{~W} / \mathrm{Hz}$ was added up-to $30000 \mathrm{~W} / \mathrm{Hz}$ subsequently. We recorded that the signal to noise ratio decreases to $0.78 \mathrm{~dB}$. We notice that as the noise was added in the system the signal strength decreases thereby causing the eye diagram to close gradual and signal is lost. The signal to noise ratio drops to $0.17 d B$ this shows that noise is notice more in the system, but there is still a little tradeoff of signal.

At $0.009 \mathrm{~km}$ noise were added, the vertical axis of the received eye diagram was recorded and the different was used for analysis. We observe that at $016 \mathrm{~dB}$ a noise of $3000 \mathrm{~W} / \mathrm{Hz}$ was added up-to $30000 \mathrm{~W} / \mathrm{Hz}$ subsequently. We recorded that the signal to noise ratio decreases to $0.6 \mathrm{~dB}$.

From our observations in Fig 3 (a) $(a=0.01 \mathrm{~km}, \mathrm{~b}=0.3 \mathrm{~km}$ and $\mathrm{c}=0.5 \mathrm{~km})$ graph and (b) $(\mathrm{a}=0.001 \mathrm{~km}, \mathrm{~b}=0.005 \mathrm{~km}$ and $\mathrm{c}=0.009 \mathrm{~km})$ graph, the signal to noise decrease as noise is increase in the system thereby reducing the effect of good signal in the communication set-up. This shows that noise introduced in the system can cause damage in the received signal and affect the system performance.

Fig.4 (a) from excel software, displays the eye diagram using the BER analyzer data within the range of $0.001 \mathrm{~km}$ which is extended to $0.9 \mathrm{~km}$ at the FSO channel range until error starts to occur. We have observed that the eye-opening is due to the optimization of different component used in the system. Above $0.9 \mathrm{~m}$ error was noticed in the signals, the eye starts to close. The BER realize as the system transmits data is less than $0.1 \%$. This shows a better performance in our system throughput. At (b) display the acceptable eye diagram of the signal transmitted from the OptiSystem software tool when noise was not added. At (c) from excel software, displays the closing of the eye diagram as noises were added from the optical detector such as Amplified signal emission (ASE) noise, distribution (Gaussian), thermal and shot noise using the same range of $0.001 \mathrm{~km}$ to $0.9 \mathrm{~m}$. This shows that signals can be corrupted or affected when noise is added to the system. Errors are observed within the channel as the signal is been transmitted thereby causing distortion in the system performance. At (d) display the eye diagram of the signal transmitted directly from the OptiSystem software tool when electronic noise was added. We have seen that both OptiSystem software and excel software present the same information. Acceptable eye diagram are displayed for good signal transmission and when noise were added there is signal change which is not acceptable.

\section{CONCLUSION}

We have discussed the importance of signal strength and the effect of signal failures in a communication system using OptiSystem software tool. FSOL optimization in VLC system is studied for improved system performance using OptiSystem software package. The acceptable eye diagram is used to detect the improved signal using a BER analyzer. The designed component were optimized for performance throughput within the sysstem. Noise were added and removed in the component for analysis on how the channel react to system transmission. The received eye diagram vertical axis difference is used to check the quantity of noise ratio performance, displaying the problem encountered. The accepted eye diagram result guarantees multi-mobile users of shared signals within the area of transmission which will improve and enhance the system throughput. We have observed that as noise was added in the system it affect the signal performance, this can be seen when the eye diagram starts to close. We notice that the different range (distances) at the FSO link can also affect the signal performance in the system as they keep on changing. Furthermore, research on how to improve, develop and implement this project is highly required as it will solve the problem of data congestion in the FSO link and upgrades communication technology system innovative and close the gap of signal failures in network connectivity.

\section{REFERENCE}

[1] R. Li, T. Chen, L. Fan and A. Dang, "Performance analysis of a multiuser dual-hop amplify-and-forward relay system with FSO/RF links," IEEE/OSA Journal of Optical 
Communications and Networking, vol. 11, no. 7, pp. 362370, July 2019.

[2] J. Chen et al., "Free-space communication turbulence compensation by optical phase conjugation," in IEEE Photonics Journal, vol. 12, no. 5, pp. 1-11, Oct. 2020.

[3] X. Zhao, L. Zhu, S. Fu, C. Liu, M. Tang and D. Liu, "Dualband accelerating beams enabled full duplex free-space optical interconnection," in IEEE Journal of Selected Topics in Quantum Electronics, vol. 27, no. 1, pp. 1-7, Jan.-Feb. 2021.

[4] H. Song et al., "Experimental mitigation of atmospheric turbulence effect using pre-signal combining for uni- and bi-directional free-space optical links with two 100-Gbit/s OAM-multiplexed channels," in Journal of Lightwave Technology, vol. 38, no. 1, pp. 82-89, 1 Jan. 1, 2020.

[5] Y. Hong, W. Shin and S. Han, "Performance of scintillation mitigation for linear polarization shift on-off keying transmission in free-space optical communications," in IEEE Access, vol. 8, pp. 128954128960, 2020.

[6] S. Liverman, H. Bialek, A. Natarajan and A. X. Wang, "VCSEL array-based gigabit free-space optical femtocell communication," in Journal of Lightwave Technology, vol. 38, no. 7, pp. 1659-1667, 1 April1, 2020.

[7] C. E. Ngene and T. Shongwe, "A sensor for monitoring the lifespan of color-LEDs in traffic lights," Proc. International Conference on Advances in Big Data, Computing and Data Communication Systems (icABCD), Winterton, South Africa, 2019, pp. 1-5.

[8] C. E. Ngene, P. Thakur and G. Singh, "Visible light communication-LEDs illuminations for smart homes," Proc. International Conference on Artificial Intelligence, Big Data, Computing and Data Communication Systems (icABCD), Durban, South Africa, 2020, pp. 1-5.

[9] C. E. Ngene, K. E. Adetunji, and T. Shongwe, "Development of an IR-based device for wireless communication in community health centres", Proc. International Conference on Intelligent and Innovative Computing Applications (ICONIC), Plaine Magnien, 2018, pp. 1-5.

[10] C. E. Ngene, P. Thakur, and G. Singh, "Power allocation techniques for visible light — non orthogonal multiple access communication systems" Ed. Mohammed Usman, Mohd Dilshad Ansari, and Mohammad Wajid, "Enabling Technologies for Next Generation Wireless Communications" CRC Press: Taylor and Francis, 2021.

[11] J. Zhang, R. Li, Z. Gao and A. Dang, "Ergodicity of phase fluctuations for free-space optical link in atmospheric turbulence," in IEEE Photonics Technology Letters, vol. 31, no. 5, pp. 377-380, 1 March1, 2019.

[12] H. Safi, A. Dargahi, J. Cheng and M. Safari, "Analytical channel model and link design optimization for ground-toHAP free-space optical communications," in Journal of Lightwave Technology, vol. 38, no. 18, pp. 5036-5047, 15 Sept.15, 2020.

[13] Y. Tu, S. Cui, K. Zhou and D. Liu, "Phase alignment with minimum complexity for equal gain combining in multiaperture free-space digital coherent optical communication receivers," in IEEE Photonics Journal, vol. 12, no. 2, pp. 1-10, April 2020.
[14] H. Kashif, M. N. Khan and A. Altalbe, "Hybrid opticalradio transmission system link quality: link budget analysis," in IEEE Access, vol. 8, pp. 65983-65992, 2020.

[15] C. Li, X. Huang, H. Lu, Y. Huang, Q. Huang and S. Tu, "A WDM PAM4 FSO-UWOC integrated system with a channel capacity of $100 \mathrm{~Gb} / \mathrm{s}$," in Journal of Lightwave Technology, vol. 38, no. 7, pp. 1766-1776, 1 April1, 2020.

[16] A. Lorences-Riesgo et al., "200 G outdoor free-spaceoptics link using a single-photodiode receiver," in Journal of Lightwave Technology, vol. 38, no. 2, pp. 394-400, 15 Jan.15, 2020.

[17] X. Li, X. Zhao, P. Zhang and S. Tong, "BER performance of FSO communication system with differential signaling over correlated atmospheric turbulence fading," in China Communications, vol. 17, no. 4, pp. 51-65, April 2020.

[18] B. Schrenk, D. Milovančev, N. Vokić, H. Hübel and F. Karinou, "Face-to-face EML transceiver tandem for fullduplex analogue radio-over-air," in Journal of Lightwave Technology, vol. 38, no. 11, pp. 2976-2983, 1 June1, 2020.

[19] J. Wang, S. Tannaz, C. Cai, A. Aliabadi, E. Mostafapour and W. Zhang, "Performance analysis of wireless adaptive incremental networks under strong FSO link turbulence conditions," in IEEE Access, vol. 8, pp. 12290-12299, 2020.

[20] M. Z. Chowdhury, M. K. Hasan, M. Shahjalal, M. T. Hossan and Y. M. Jang, "Optical wireless hybrid networks: trends, opportunities, challenges, and research directions," in IEEE Communications Surveys \& Tutorials, vol. 22, no. 2, pp. 930-966, Secondquarter 2020.

[21] E. Saleh Altubaishi and K. Alhamawi, "Capacity analysis of hybrid AF multi-hop FSO/RF system under pointing errors and weather effects," in IEEE Photonics Technology Letters, vol. 31, no. 15, pp. 1304-1307, 1 Aug.1, 2019.

[22] A. S. Hamza, J. S. Deogun and D. R. Alexander, "Evolution of data centers: a critical analysis of standards and challenges for FSO links," In IEEE Conference on Standards for Communications and Networking (CSCN), Tokyo, 2015, pp. 100-105.

[23] A. S. Hamza, J. S. Deogun and D. R. Alexander, "Classification framework for free space optical communication links and systems," in IEEE Communications Surveys \& Tutorials, vol. 21, no. 2, pp. 1346-1382, Secondquarter 2019.

[24] J. L. Johnson, F. Jasman, R. J. Green, and M. S. Leeson, 2014, "Recent advances in underwater optical wireless communications," Underwater Technology, vol. 32, no. 3, pp. 167-175.

[25] Technology demonstration missions, "Laser communications relay demonstration (LCRD)," D. J. Israel, B. L. Edwards and J. W. Staren, "Laser Communications Relay Demonstration (LCRD) update and the path towards optical relay operations," in IEEE Aerospace Conference, Big Sky, MT, 2017, pp. 1-6.

[26] M. A. Khalighi and M. Uysal, "Survey on free space optical communication: a communication theory Perspective," In IEEE Communications Surveys \& Tutorials, vol. 16, no. 4, pp. 2231-2258, Fourth quarter 2014.

[27] X. Deng, Y. Wu, K. Arulandu, G. Zhou and J. M. G. Linnartz, "Performance comparison for illumination and visible light communication system using buck 
converters," In IEEE Globecom Workshops (GC Wkshps), Austin, TX, 2014, pp. 547-552.

[28] D. Wu, Z. Ghassemlooy, W. Zhong and C. Chen, "Cellular indoor OWC systems with an optimal lambertian order and a handover algorithm," Proc, $7^{\text {th }}$ International Symposium on Telecommunications (IST'2014), Tehran, 2014, pp. 777782.

[29] M. Rahaim and T. D. C. Little, "SINR analysis and cell zooming with constant illumination for indoor VLC networks," 2nd International Workshop on Optical Wireless Communications (IWOW), Newcastle upon Tyne, 2013, pp. 20-24.

[30] I. Din and H. Kim, "Energy-efficient brightness control and data transmission for visible light communication," in IEEE Photonics Technology Letters. vol. 26, no. 8, pp. 781-784, 2014.

[31] S. O. Mohammadi, S. Mozzaffari and M. Mahdi Shahidi, "Simulation of a transmission system to compensate dispersion in an optical fiber by chirp gratings." International Journal of the Physical Sciences, vol. 6, no. 32, pp. 7354 - 7360, 2 December 2011.

[32] I. E. Lee, G. C. Chung, W. L. Pang, S. S Anas and M. Y. Cheong, "Design of a hybrid free space optical and visible light communication system for indoor wireless data broadcasting" in Journal of Physics: Conference Series., vol. 1, pp.1-11, 2020.

[33] G. G. Lema, "Free space optics communication system design using iterative optimization" in Journal of Optical Communications., vol.1, pp.1-12, 2020. 
Figures

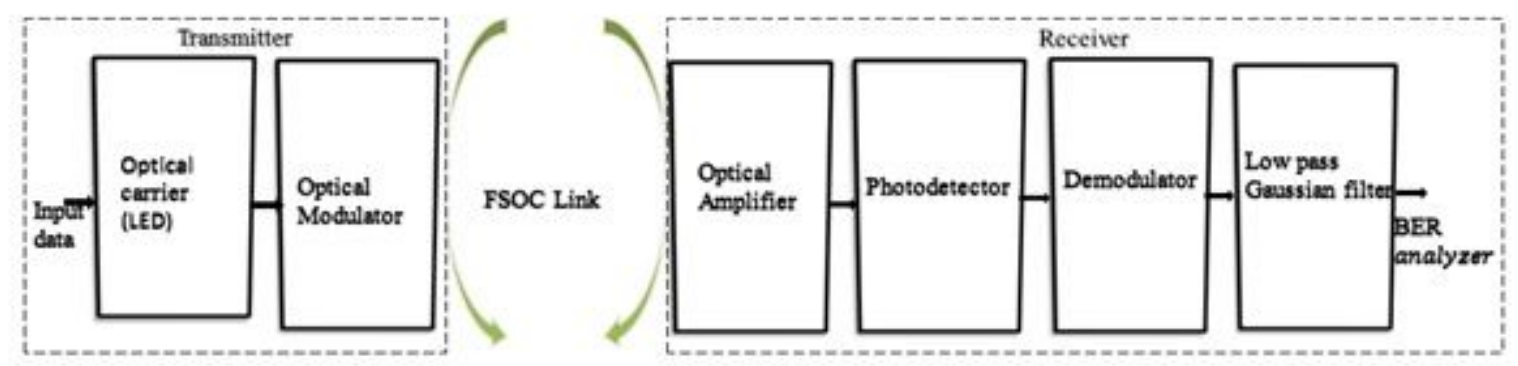

Figure 1

A block diagram of a free-space optical link optimization in visible light communication system.

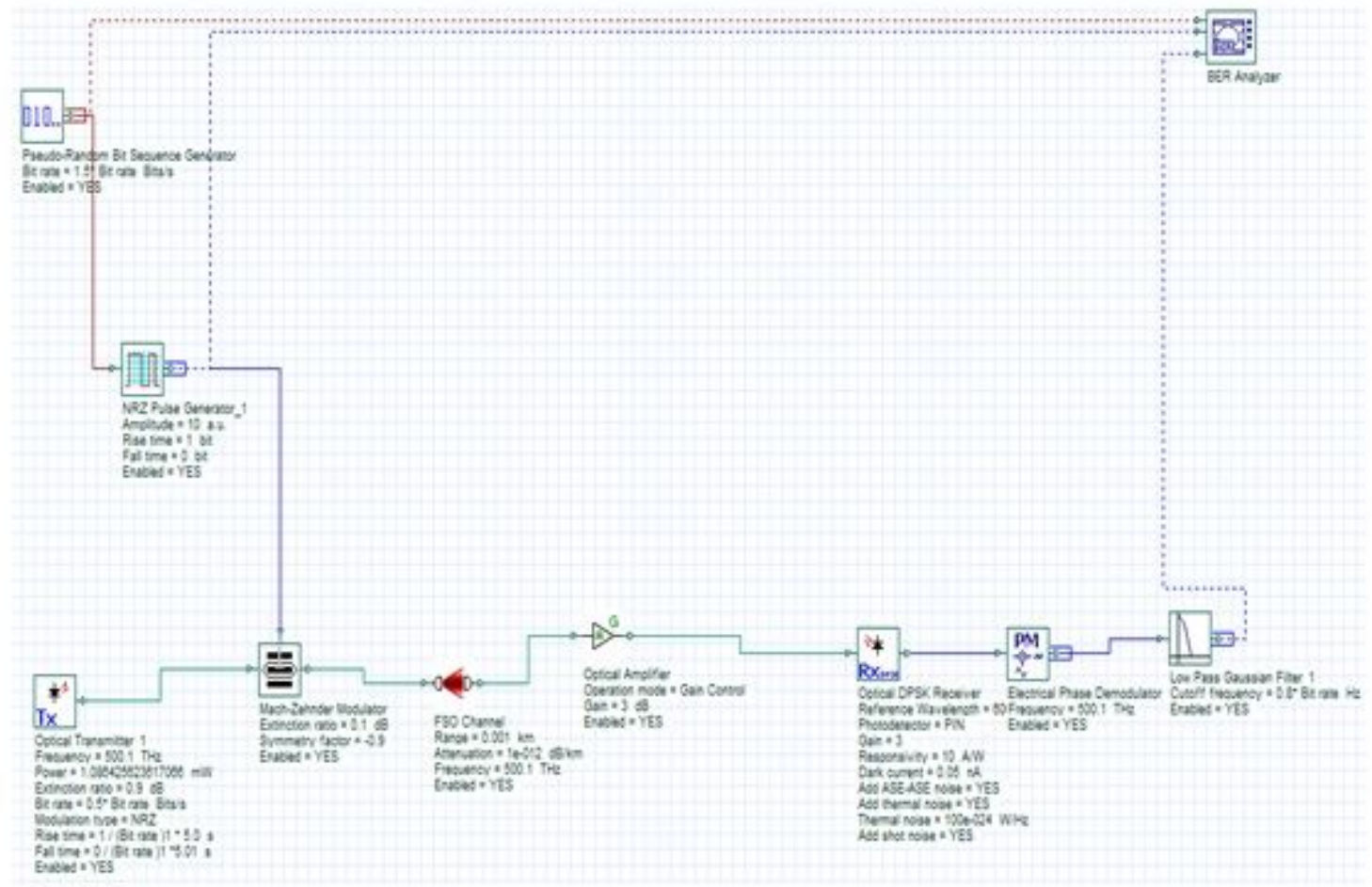

Figure 2

The system model of a free-space optical link optimization in visible light communication system. 


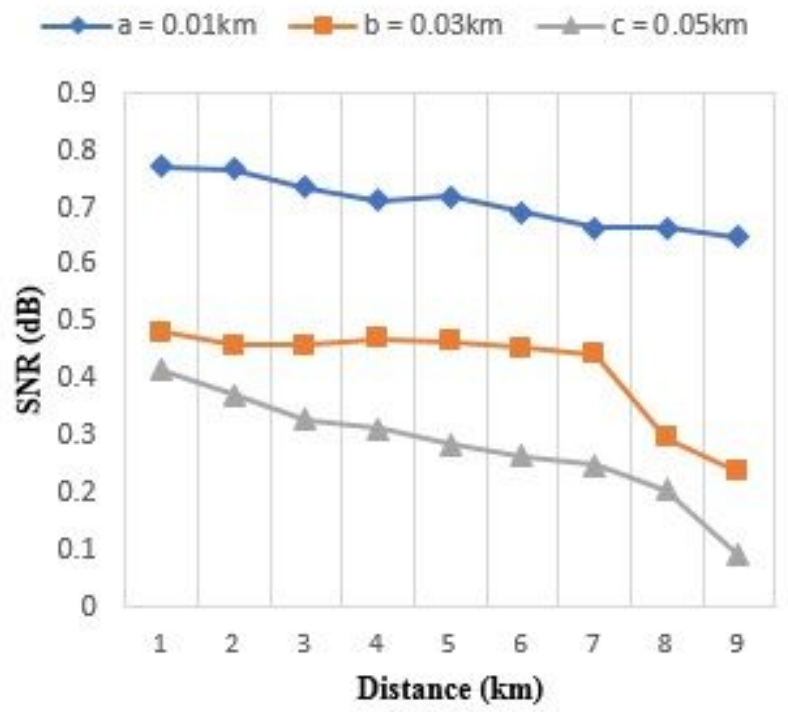

(a)

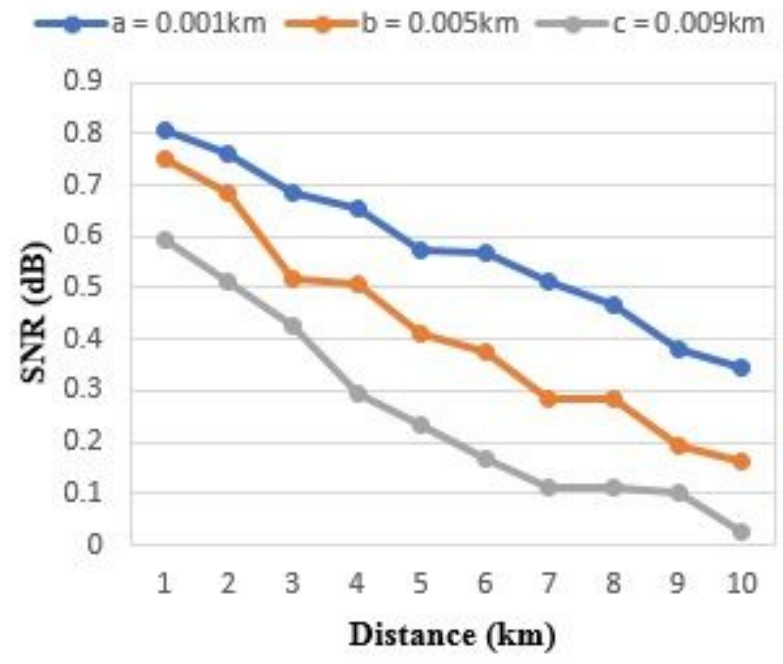

(b)

\section{Figure 3}

Variation on the SNR (in dB) with distance in the indoor noise added ranges for (a) optical amplifier (b) optical detector. 


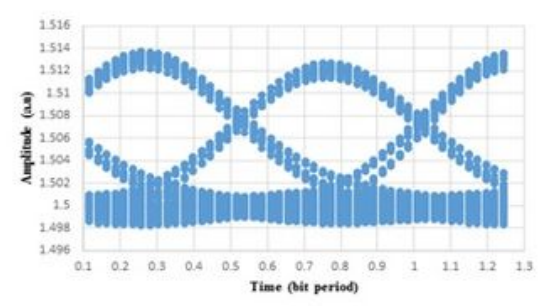

(a)

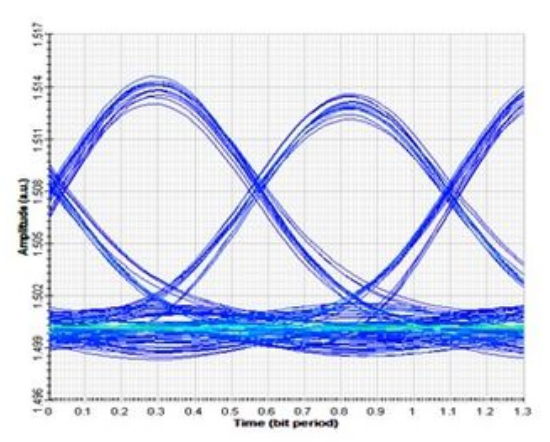

(b)

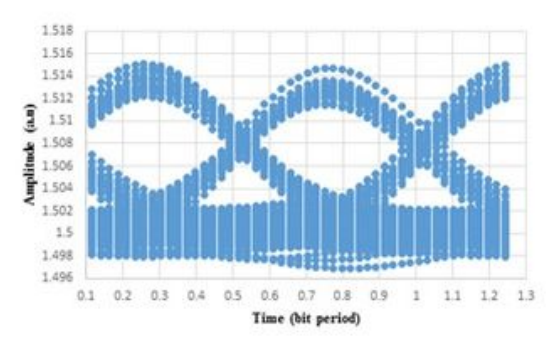

(c)

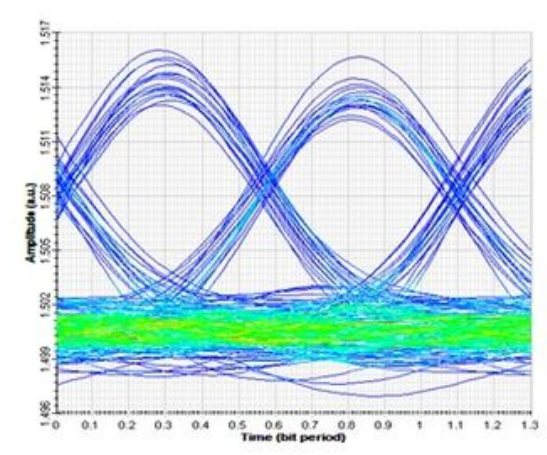

(d)

\section{Figure 4}

The eye diagram using OptiSystem and excel software (a) acceptable eye diagram from excel without noise, (b) acceptable eye diagram from OptiSystem without noise (c) eye diagram with noise from excel (d) eye diagram from OptiSystem with noise. 